\title{
The Pleiotropic Effects of Statins in the Prevention of Atherosclerosis
}

\author{
Editorial to: "Simvastatin Suppresses Apoptosis in Vulnerable Atherosclerotic Plaques \\ Through Regulating the Expression of p53, Bcl-2 en Bcl-xL" by Weiwei Qin et al.
}

\author{
Wei Kong • Yi Zhu
}

Published online: 5 November 2011

(C) Springer Science+Business Media, LLC 2011

Statins, as the biggest selling class of pharmaceutical compounds, have become the cornerstone of medical treatment for hypercholesterolemia and have led to a marked reduction in morbidity and mortality from adverse cardiovascular diseases (CVD). They were initially isolated and identified as metabolites of red rice yeast $[1,2]$ to inhibit the rate-limiting step of cholesterol biosynthesis, the conversion of HMG-CoA to L-mevalonic acid, through blocking the activity of 3-hydroxy-3-methylglutaryl coenzyme A reductase (HMG CoA-reductase) [3]. This leads to decreased hepatic cholesterol biosynthesis, and feedback increased hepatic expression of low-density lipoprotein (LDL) receptor, which results in enhanced LDL-cholesterol clearance in the circulation. Because plasma LDL cholesterol level is strongly associated with coronary heart disease, it has been generally assumed that the beneficial effects underlying statin therapy are entirely due to cholesterol reduction. Interestingly, both experimental and clinical evidence reveal the overall beneficial effects of statins extend beyond those obtained by simply lowering serum cholesterol levels [4-6]. Thus, statins are believed to have "pleiotropic effects," a term used to collectively refer to the beneficial effects of statins that are independent of cholesterol levels. Such pleiotropic effects on the vessel wall have been suggested to be multifaceted, including attenuation of vascular inflammation, improved endothelial cell function, Increased circulating endothelial progenitor cells and enhancement of function, decreased vascular smooth muscle cell (VSMC)

W. Kong $\cdot$ Y. Zhu $(\bowtie)$

Department of Physiology and Pathophysiology,

Peking University Health Sciences Center,

Beijing 100191, China

e-mail: zhuyi@bjmu.edu.cn proliferation and migration, stabilization of atherosclerotic plaque, and inhibition of platelet aggregation [7-9].

With respect to the potential mechanism of statins' pleiotropic effects, there are diverse targets and pathways. First, by inhibiting HMG-CoA reductase, statins could also inhibit the synthesis of downstream isoprenoid intermediates, such as farnesylpyrophosphate (FPP) and geranylgeranylpyrophosphate (GGPP) [10]. These two isoprenoids are both used to provide intracellular proteins with lipophilic attachments to the cell membrane [11]. Ras and Rho families of small $G$ proteins are important components of second messenger systems in vascular inflammation, hypertrophy, and in promoting vascular remodeling in disease states. Both Ras and Rho family proteins are known to require these lipophilic attachments for appropriate localization and activity within the membrane $[12,13]$. Ras family of small G proteins need FPP to be farnesylated in the membrane attachment and functional activity, and GGPP performs the geranylgeranylation for Rho and its isoforms $[14,15]$. These post-translational modifications can be inhibited by statins. Thus, in addition to cholesterol lowering, the inhibition of these intracellular isoprenoiddependent proteins may contribute to some of the biological effects of statins.

Secondly, statins promote the upregulation of endothelial nitric oxide synthase (sNOS) and improve pathophysiologic response, including inhibition of vasoconstriction and promotion of reendothelialization. The activation of eNOS by statins is mediated through the phosphatidylinositol-3 kinase (PI3K)-Akt pathway [16] and AMP-activated protein kinase (AMPK) $[17,18]$. Laufs et al also showed that statins can prevent the downregulation of eNOS expression and activity induced by oxLDL or hypoxia $[19,20]$.

Pleiotropic effects of statins may also act through activation of peroxisome proliferator-activated receptors 
(PPARs). Statins are reported to activate PPAR- $\gamma$ and inhibit LPS-induced cytokine expression in macrophages [21]. These findings are supported by data demonstrating that statins stabilize atherosclerotic plaques through the activation of PPAR- $\gamma$ and that combined administration of simvastatin with PPAR- $\gamma$ agonists elicits additive effects on atherosclerotic plaque regression [22-24].

In this issue, Qin and colleagues [25] found that simvastatin did not affect the size of atherosclerotic lesion in apoE-/mice, but decreased the area of lipid core and increased the amounts of macrophages and VSMCs in atherosclerotic plaques of apoE- $-/-$ mice by regulating the expression of apoptosis-related proteins, such as $\mathrm{p} 53, \mathrm{Bcl}-2$ and $\mathrm{Bcl}-\mathrm{xL}$. This study reveals a novel mechanism of beneficial effect of statins. Atherosclerotic plaque rupture is a major cause of acute ischemic episodes in both the cardiac and peripheral vasculature. Vulnerable atherosclerotic plaques contain an abundance of macrophages that express matrix metalloproteinases (MMPs) and tissue factor (TF) [26, 27]. Aikawa et al reported that cerivastatin could decrease macrophage growth and subsequent expression of MMPs and TF both in vitro and in vivo in a hypercholesterolemic rabbit model [26]. Indeed, Crisby et al studied the effects of pravastatin which significantly reduced MMP-2 immunoreactivity on human carotid plaque composition [27]. On the other hand, accumulation of lipids in the atherosclerotic plaque causes both mechanical and biologic instability of the plaque itself. Lipid lowering through statin treatment can promote plaque stability while attenuating bioactive lipids-induced oxidative stress and inflammatory response [28]. Although the expression of MMPs and TF was not measured in this study, the authors found that simvastatin downregulated the expression of vascular cell adhesion molecule-1 (VCAM-1) by both inhibition of nuclear factor kappa B activation and suppression of the expression of the receptor for advanced glycation end products. This result supports the inhibitory effect of statin on oxidative stress and inflammatory response in the plaque [25].

Furthermore, the results from Qin et al. [25] show that no significant differences in levels of serum LDLcholesterol and HDL-cholesterol were found between the simvastatin treated group and the controls, supporting the notion that the clinical benefits of statin therapy may be independent of LDL-cholesterol reduction. Recently, the JUPITER clinical trial showed that statins could be beneficial in the primary prevention of CVD in patients with elevated high-sensitivity C-reactive protein (hsCRP), but relatively low LDL-cholesterol levels [29]. JUPITER evaluated the effect of rosuvastatin $(20 \mathrm{mg} /$ day $)$, and found that rosuvastatin was of particular benefit if low concentrations of both LDL-cholesterol and CRPs were achieved [29]. Another clinical trial from the multicenter coronary atherosclerosis study measuring the effects of rosuvastatin using intravascular ultrasound in Japanese subjects (COSMOS) also confirmed this conclusion [30].

In conclusion, the pleiotropic effects of statins in CVD are secondary to a variety of processes at both molecular and cellular levels. Some of these beneficial effects include improvement in endothelial function, decreased vascular inflammation, inhibition of VSMC migration and proliferation, increased apoptosis of macrophages and VSMCs in the atherosclerotic plaques and increased stability of the plaque. However, statin pleiotropy is still an evolving concept and clinical studies have yet to show how many of the benefits of statins are non-LDL dependent. Part of the reason is due to the lack of a definitive biomarker for statin pleiotropy [8]. With the molecular insights obtained from basic studies regarding the signaling pathways involved in statin pleiotropy, it may be possible in the near future to actually define the clinical benefits of statin therapy attributed to non-LDL effects.

\section{References}

1. Alberts AW, Chen J, Kuron G, et al. Mevinolin: a highly potent competitive inhibitor of hydroxymethylglutaryl-coenzyme A reductase and a cholesterol-lowering agent. Proc Natl Acad Sci USA. 1980;77:3957-61.

2. Alberts AW. Discovery, biochemistry and biology of lovastatin. Am J Cardiol. 1988;62:10J-5J.

3. Istvan ES, Deisenhofer J. Structural mechanism for statin inhibition of HMG-CoA reductase. Science. 2001;292:1160-4.

4. Group Heart Protection Study Collaborative. MRC/BHF Heart Protection Study of cholesterol lowering with simvastatin in 20536 high-risk individuals: a randomised placebo-controlled trial. Lancet. 2002;360:7-22.

5. Almuti K, Rimawi R, Spevack D, et al. Effects of statins beyond lipid lowering: potential for clinical benefits. Int $\mathrm{J}$ Cardiol. 2006;109:7-15.

6. Liao JK. Clinical implications for statin pleiotropy. Curr Opin Lipidol. 2005;16:624-9.

7. Mihos CG, Salas MJ, Santana O. The pleiotropic effects of the hydroxy-methyl-glutaryl-CoA reductase inhibitors in cardiovascular disease: a comprehensive review. Cardiol Rev. 2010;18:298304.

8. Zhou Q, Liao JK. Pleiotropic effects of statins.-basic research and clinical perspectives. Circ J. 2010;74:818-26.

9. Sadowitz B, Maier KG, Gahtan V. Basic science review: statin therapy_-Part I: the pleiotropic effects of statins in cardiovascular disease. Vasc Endovasc Surg. 2010;44:241-51.

10. Goldstein JL, Brown MS. Regulation of the mevalonate pathway. Nature. 1990;343:425-30.

11. Van Aelst L, D'Souza-Schorey C. Rho GTPases and signaling networks. Genes Dev. 1997;11:2295-322.

12. Hall A. G proteins and small GTPases: distant relatives keep in touch. Science. 1998;280:2074-5.

13. Adamson P, Marshall CJ, Hall A, Tilbrook PA. Post-translational modifications of p21rho proteins. J Biol Chem. 1992;267:200338 .

14. Argmann CA, Edwards JY, Sawyez CG, et al. Regulation of macrophage cholesterol efflux through hydroxymethylglutarylCoA reductase inhibition. J Biol Chem. 2005;280:22212-21. 
15. Casey PJ. Protein lipidation in cell signaling. Science. 1995;268:2215.

16. Kureishi Y, Luo Z, Shiojima I, et al. The HMG-CoA reductase inhibitor simvastatin activates the protein kinase Akt and promotes angiogenesis in normocholesterolemic animals. Nat Med. 2000;6:1004-10.

17. Sun W, Lee T-S, Zhu MJ, et al. Statins activate AMP-activated kinase in vitro and in vivo. Circulation. 2006;114:2655-62.

18. Li X, Han Y, Pang W, et al. AMP-activated protein kinase promotes the differentiation of endothelial progenitor cells via eNOS-derived NO. Arterio Thromb Vasc Biol. 2008;28:1789-95.

19. Laufs U, La Fata V, Plutzky J, et al. Upregulation of endothelial nitric oxide synthase by HMG CoA reductase inhibitors. Circulation. 1998;97:1129-35.

20. Laufs U, La Fata V, Liao JK. Inhibition of 3-hydroxy-3methylglutaryl (HMG)-CoA reductase blocks hypoxia-mediated down-regulation of endothelial nitric oxide synthase. J Biol Chem. 1997;272:31725-9.

21. Yano M, Matsumura $T$, Senokuchi $T$, et al. Statins activate peroxisome proliferator-activated receptor gamma through extracellular signal-regulated kinase $1 / 2$ and p38 mitogen-activated protein kinase-dependent cyclooxygenase- 2 expression in macrophages. Circ Res. 2007;100:1442-51.

22. Sugamura K, Sugiyama S, Matsuzawa Y, et al. Benefit of adding pioglitazone to successful statin therapy in nondiabetic patients with coronary artery disease. Circ J. 2008;72:1193-7.

23. Zelvyte I, Dominaitiene R, Crisby M, Janciauskiene S. Modulation of inflammatory mediators and PPARgamma and NFkappaB expression by pravastatin in response to lipoproteins in human monocytes in vitro. Pharmacol Res. 2002;45:147-54.
24. Corti R, Osende JI, Fallon JT, et al. The selective peroxisomal proliferator-activated receptor-gamma agonist has an additive effect on plaque regression in combination with simvastatin in experimental atherosclerosis: in vivo study by high-resolution magnetic resonance imaging. J Am Coll Cardiol. 2004;43:464-73.

25. Qin W, Lu Y, Zhan C, et al. Simvastatin suppresses apoptosis in vulnerable atherosclerotic plaques through regulating the expression of p53, Bcl-2 and Bcl-xL. Cardiovasc Drugs Ther 2012;26: this issue

26. Aikawa M, Rabkin E, Sugiyama S, et al. An HMG-CoA reductase inhibitor, cerivastatin, suppresses growth of macrophages expressing matrix metalloproteinases and tissue factor in vivo and in vitro. Circulation. 2001;103:276-83.

27. Crisby M, Nordin-Fredriksson G, Shah PK, et al. Pravastatin treatment increases collagen content and decreases lipid content, inflammation, metalloproteinases, and cell death in human carotid plaques: implications for plaque stabilization. Circulation. 2001;103:926-33.

28. Koh KK. Effects of statins on vascular wall: vasomotor function, inflammation, and plaque stability. Cardiovasc Res. 2000;47:64857.

29. Ridker PM, Danielson E, Fonseca FA, et al. Reduction in Creactive protein and LDL cholesterol and cardiovascular event rates after initiation of rosuvastatin: a prospective study of the JUPITER trial. Lancet. 2009;373:1175-82.

30. Takayama T, Hiro T, Yamagishi M, et al. Effect of rosuvastatin on coronary atheroma in stable coronary artery disease: Multicenter coronary atherosclerosis study measuring effects of rosuvastatin using intravascular ultrasound in Japanese subjects (COSMOS). Circ J. 2009;73:2110-7. 